Virginia Commonwealth University

vCU Scholars Compass

1996

\title{
Oxidation states and magnetism of Fe nanoparticles prepared by a laser evaporation technique
}

B.J.Jönsson

Royal Institute of Technology

T. Turkki

Royal Institution of Technology

V. Ström

Royal Institution of Technology

M. S. El-Shall

Royal Institution of Technology

K.V.Rao

Royal Institution of Technology

Follow this and additional works at: http://scholarscompass.vcu.edu/chem_pubs

Part of the Chemistry Commons

Jonsson, B. J., Turkki, T., Strom, V., et al. Oxidation states and magnetism of Fe nanoparticles prepared by a laser evaporation technique. Journal of Applied Physics 79, 5063 (1996). Copyright (C) 1996 AIP Publishing LLC.

\section{Downloaded from}

http://scholarscompass.vcu.edu/chem_pubs/41

This Article is brought to you for free and open access by the Dept. of Chemistry at VCU Scholars Compass. It has been accepted for inclusion in Chemistry Publications by an authorized administrator of VCU Scholars Compass. For more information, please contact libcompass@vcu.edu. 


\title{
Oxidation states and magnetism of Fe nanoparticles prepared by a laser evaporation technique
}

\author{
B. J. Jönsson, T. Turkki, V. Ström, M. S. El-Shall,a) and K. V. Rao \\ Royal Institute of Technology, Department of Condensed Matter Physics, 10044 Stockholm, Sweden
}

\begin{abstract}
Nanoparticles of iron and iron oxide have been prepared in a thermal diffusion cloud chamber using pulsed laser evaporation. SEM/TEM studies of these particles reveal a size distribution with a mean diameter of about $60 \AA$. This is consistent with the mean particle size estimated from the magnetic data. The oxidation levels of these nanoparticles prepared at different partial oxygen pressures were investigated using FTIR. All the samples are found to exhibit superparamagnetism with blocking temperatures ranging from $50 \mathrm{~K}$ to above room temperature. Magnetic anisotropy constants are calculated from the frequency dependence of the blocking temperatures are found to be one quarter of magnitude higher than is known for the bulk. () 1996 American Institute of Physics. [S0021-8979(96)40108-1]
\end{abstract}

\section{INTRODUCTION}

Ultrafine or nanoparticles have been the subject of intense research in recent years. Nanoparticles have unique properties in comparison with the bulk: large surface to volume ratio, discrete electron energy levels which can be easily tuned over a wide range of compositions, etc. Magnetic nanoparticles become single domain below a certain size and exhibit superparamagnetism. Interest in magnetic nanoparticles remains high because of the wide range of potential applications, including information storage, ferrofluids, permanent magnets and pigments in paints. Iron oxide nanoparticles are also expected to have properties as high performance catalysts.

In this paper, we present the magnetic properties of iron and iron oxide nanoparticles produced by pulsed laser evaporation in different oxygen partial pressure atmosphere. The superparamagnetic properties of these nanoparticles are also presented.

\section{EXPERIMENT}

Iron oxide samples were prepared in a modified upward thermal diffusion cloud chamber. ${ }^{1,2}$ A sketch of the chamber is shown in Fig. 1. The iron target was set on the bottom plate. The chamber was filled with desired amount of oxygen and further filled with helium to 800 Torr. The top plate was cooled down to $-100{ }^{\circ} \mathrm{C}$ while the bottom plate was at room temperature. Metal was vaporized using Nd:YAG laser (532 $\mathrm{nm}, 20 \mathrm{~Hz}$, and $20 \mathrm{~mJ} / \mathrm{pulse}$ ). In the present investigations the partial oxygen pressure was varied from 0 to 500 Torr while keeping the total pressure at 800 Torr.

SEM and TEM micrographs were taken with JEOL SM 840 and JEOL 2000 EX instruments, respectively. The infrared absorption spectra were recorded on a Nicolet 750 Magna Fourier transform spectrophotomer under atmospheric pressure. dc magnetic measurements were performed using Quantum $\mathrm{MPMS}_{2}$ SQUID magnetometer. The zero field cooled measurements were performed by cooling the sample to $5 \mathrm{~K}$ at zero field and then applying $20 \mathrm{G}$ field for

\footnotetext{
a) Permanent address: Virginia Commonwealth University, Department of Chemistry, Richmond, VA 23284-2006.
}

the warming up scan. Hysteresis loops were measured at selected temperatures. Between every loop the sample was warmed up to $300 \mathrm{~K}$ and the remanence field was minimized. ac magnetic measurements were performed using a homebuilt two-position ac susceptometer with a three-coil mutual inductance bridge. The driving field was 3 Oe for all measurements and the frequency was varied from $4 \mathrm{~Hz}$ to $4 \mathrm{kHz}$.

\section{RESULTS AND DISCUSSION}

For brevity we label the samples Fe- $0, \mathrm{Fe}-10 \ldots$ allowing the number to denote the partial oxygen pressure, in Torr, during preparation. The color of the samples goes from very dark brown, almost black (the color of $\mathrm{FeO}$ and $\mathrm{Fe}_{3} \mathrm{O}_{4}$ ) to reddish brown (the color of $\gamma-\mathrm{Fe}_{2} \mathrm{O}_{3}$ ) with increasing oxygen pressure. TEM bright field images (Fig. 2) show the samples to consist of very small particles with mean diameter of about $60 \AA$ A. Some larger particles with sizes $50-500 \mathrm{~nm}$ are also present. No significant difference in particle size distribution is observed between different samples. The size of the particles is found to be controlled by the total pressure, temperature gradient, and the partial pressure of the metal vapor. These parameters were kept the same for every preparation. The diffuse TEM electron diffraction patterns indicate the presence of $\mathrm{FeO}, \mathrm{Fe}_{3} \mathrm{O}_{4}$, and $\gamma-\mathrm{Fe}_{2} \mathrm{O}_{3}$ phases in all samples.

Because of the high surface to volume ratio the activity at the surface of the nanoparticles would be significantly different from that of the bulk. From the Fourier transformed, FTIR absorption data we find that in these particles the chemisorption of $\mathrm{CO}_{2}$ increases with the partial oxygen pressure. At oxygen partial pressures below 200 Torr the samples are found to consist mainly of $\mathrm{Fe}_{3} \mathrm{O}_{4}$ with some $\gamma-\mathrm{Fe}_{2} \mathrm{O}_{3}$ contamination. The $\gamma-\mathrm{Fe}_{2} \mathrm{O}_{3}$ peak at $450 \mathrm{~cm}^{-1}$ is barely visible. At higher partial oxygen pressures the sample stoichiometry appears to be close to the $\gamma-\mathrm{Fe}_{2} \mathrm{O}_{3}$ form.

The iron and iron oxide particles prepared in this study, have sizes within the single domain particle regime (about $200 \AA$ for $\mathrm{Fe}$ ). Their magnetic behavior is well described in terms of superparamagnetism. For a superparamagnet the relaxation time $\tau$ is given by. ${ }^{3}$

$$
\tau=\tau_{0} e^{K V / k_{B} T}
$$




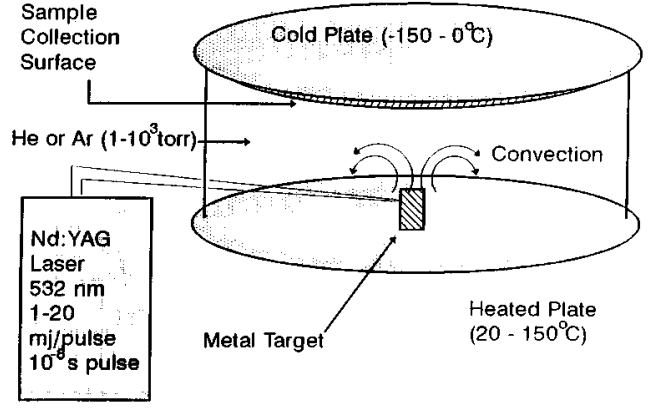

FIG. 1. A sketch of the diffusion cloud chamber.

where $K$ and $V$ are the anisotropy constant and the particle volume, respectively, and $\tau_{0}$ is of the order of $10^{-9} \mathrm{~s}$. The superparamagnetic behavior is observed above the blocking temperature $T_{B}$. Below $T_{B}$ the particles are magnetically frozen and hysteresis appears.

The in-phase ac susceptibility $\chi^{\prime}$ was measured for five samples prepared at different partial oxygen pressures (Fig. 3). $\chi^{\prime}$ is found to increase monotonically with temperature for the Fe-0 sample. This behavior is consistent with expected blocking temperature ${ }^{4}$ of $300 \mathrm{~K}$ for iron particles in the size range $60-80 \AA$. When the particles consist of iron oxide instead of iron, there is a dramatic change in behavior. A clear cusp is seen at about $65 \mathrm{~K}$ in sample Fe-10. Increasing the oxidation of iron, as in $\mathrm{Fe}-50$, gives rise to another cusp around $110 \mathrm{~K}$ which then dominates in Fe-200. Finally, we observe only one cusp in the Fe-500 sample.

There is a sudden increase in coercivity below the blocking temperature (Fig. 4). The coercivity does not fall to zero above $T_{B}$ as expected for a purely superparamagnetic sample. This is probably due to the existence of the few large-size particles $(50-500 \mathrm{~nm})$ seen in the TEM micrographs.

The magnetization of a paramagnet follows a Langevin function:

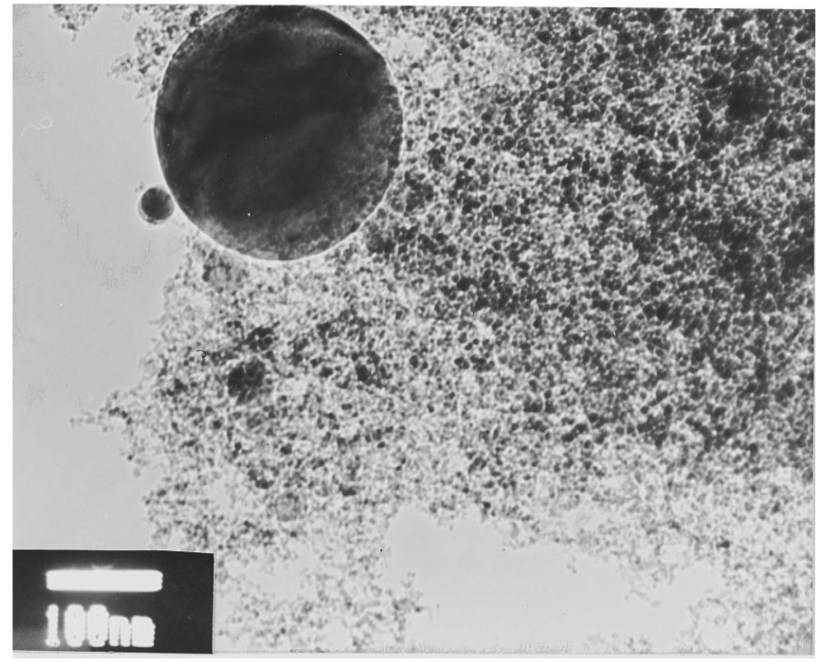

FIG. 2. TEM micrograph of the Fe-10 sample.

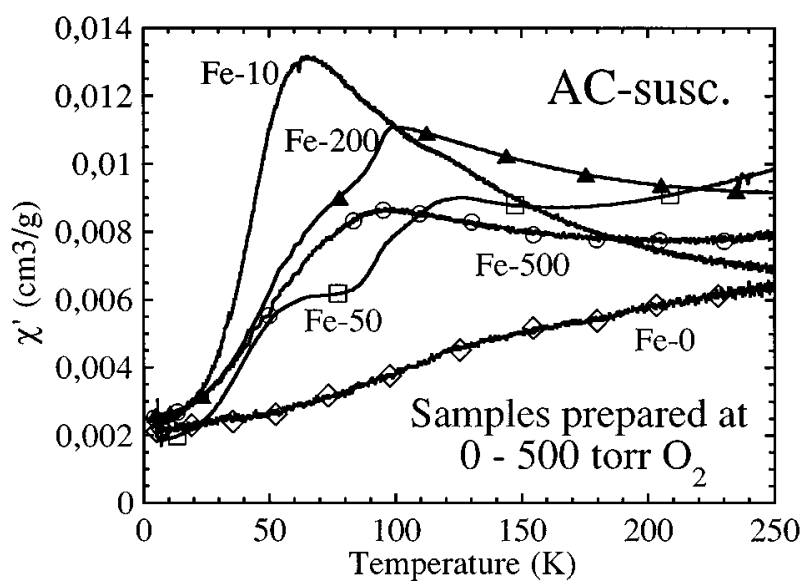

FIG. 3. In-phase ac susceptibility as a function of temperature.

$$
\frac{M}{M_{s}}=L(a)=\operatorname{coth} a-\frac{1}{a}, \quad a=\frac{\mu H}{k_{B} T} .
$$

Since the Langevin function is a linear function for small arguments, $L(a) \approx a / 3$, this will hold for superparamagnetic particles of different sizes at low fields. Thus, measuring $M / H$ loops and taking the slope around zero field gives us a means of calculating the mean-magnetic moment of our particles and therefore, a mean-particle diameter.

The mean-magnetic moment per particle for sample $\mathrm{Fe}-10$ is found to be $5100 \mu_{B}$. Assuming the dominant phase to be $\mathrm{Fe}_{3} \mathrm{O}_{4}$, which has $4.1 \mu_{B}$ per molecule, eight molecules per unit cell and a lattice constant of $8.39 \AA$, we estimate the mean-particle diameter to be around $60 \AA$, in good agreement with the TEM observations.

Knowing the mean volume of our particles we can now estimate $K_{1}$. We measured $\chi^{\prime}$ at six different frequencies between 4 and $4000 \mathrm{~Hz}$ and included a SQUID measurement with a measuring time of about $100 \mathrm{~s}$. Taking the maximum of $\chi^{\prime}$ to be the blocking temperature and plotting $1 / T_{B}$ vs $\ln (f), f$ being the driving frequency, we get a straight line (Fig. 5). The slope of this line is equal to $-k_{B}\left(\left|K_{1}\right| V\right)^{-1}$, according to Eq. (1).

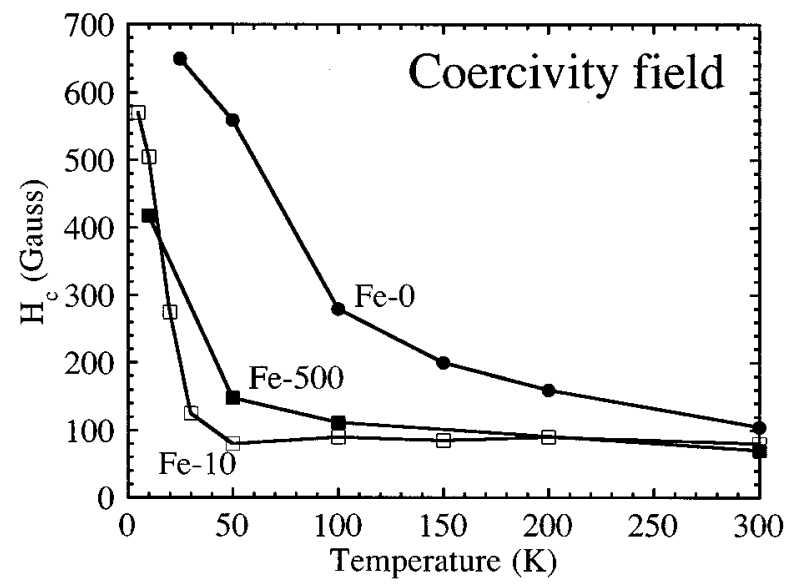

FIG. 4. Coercivity field as a function of temperature for samples showing one single cusp. 


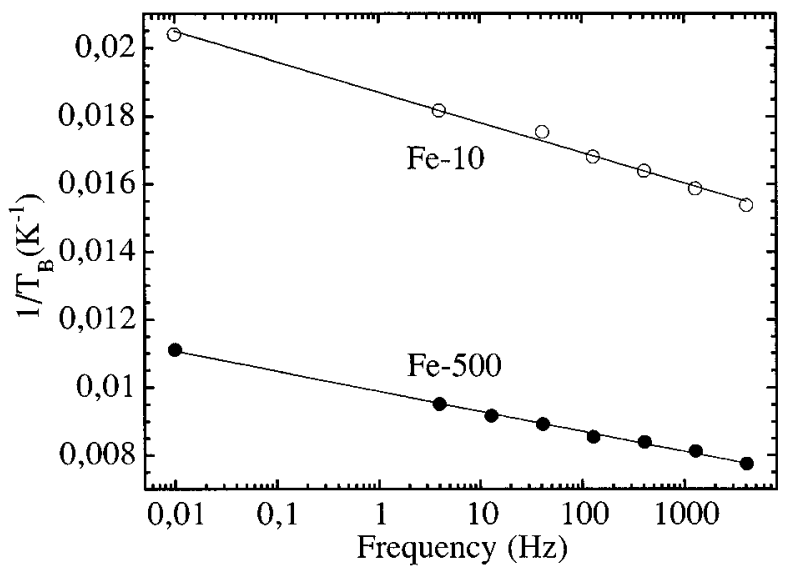

FIG. 5. Plot showing the blocking temperature as a function of measuring frequency for samples Fe-10 and Fe-500.

In the case of a cubic structure with the easy direction along $\langle 111\rangle, K_{1}$ is replaced ${ }^{5}$ by $K_{1} / 12$. We thus get an anisotropy constant $\left|K_{1}\right|=2.1 \times 10^{6} \mathrm{ergs} / \mathrm{cm}^{3}$ for sample Fe-10. If we assume the mean size to be equal in all samples we get $\left|K_{1}\right|=3.1 \times 10^{6} \mathrm{ergs} / \mathrm{cm}^{3}$ for the sample Fe-500. Those values for $\left|K_{1}\right|$ are an order of magnitude larger than the values for bulk $\mathrm{Fe}_{3} \mathrm{O}_{4}$ and $\gamma$ - $\mathrm{Fe}_{2} \mathrm{O}_{3}$, but agrees well with values reported in literature ${ }^{6}$ for small particles.

Since all samples have about the same size distribution it is the difference in composition that determines the different magnetic behavior. The sample Fe-0 has a high blocking temperature due to the anisotropy barrier being higher for iron than for its oxides. In iron the easy directions are along the cube sides $\langle 100\rangle$ and $K_{1}$ should be replaced by $K_{1} / 4$ and not $K_{1} / 12$. When the core of the nanoparticles is $\mathrm{Fe}_{3} \mathrm{O}_{4}$ the anisotropy barrier is reduced and the blocking temperature falls to about $50 \mathrm{~K}$. With increasing oxidation, the fraction of $\gamma-\mathrm{Fe}_{2} \mathrm{O}_{3}$ increases and since its anisotropy barrier is slightly higher than that for $\mathrm{Fe}_{3} \mathrm{O}_{4}$, a new cusp develops at about 110
K. As the $\gamma-\mathrm{Fe}_{2} \mathrm{O}_{3}$ fraction further increases this cusp dominates and is the only one observed in the sample Fe-500.

\section{CONCLUSIONS}

We have demonstrated a simple technique to prepare nanoparticles of iron and iron oxide. By varying the partial oxygen pressure in preparation we have been able to change the oxidation state of Fe. Particles prepared at low oxygen pressure consist mainly of $\mathrm{FeO}$ and $\mathrm{Fe}_{3} \mathrm{O}_{4}$ while those prepared at higher oxygen pressure consist of $\gamma-\mathrm{Fe}_{2} \mathrm{O}_{3}$. As a consequence the magnetic behavior changes in a rather complex way. The magnetic properties, analyzed in terms of superparamagnetism, are found to be consistent with the implications from the particle size and structure. Magnetic anisotropy constants calculated from our experimental data agree well with the expected values for small particles.

\section{ACKNOWLEDGMENTS}

It is a pleasure to acknowledge many valuable discussions with Professor Dan E. Dahlberg during his visit with us. T. Turkki is most obliged to colleagues at the Department of Chemistry at VCU for their hospitality and financial support during her stay at VCU. This research has been partially supported by the Swedish National Cluster Consortium, and NSF Grants DMR 9207433 and CHE 9311643, in the United States.

${ }^{1}$ M. S. El-Shall, W. Slack, W. Vann, D. Kane, and D. Hanley, J. Phys. Chem. 98, 3067 (1994).

${ }^{2}$ M. S. El-Shall, W. Slack, W. Vann, D. Hanley, and D. Kane, Mater. Res. Soc. Symp. Proc. 351, 369 (1994).

${ }^{3}$ L. Néel, Ann. Geophys. 5, 99 (1949).

${ }^{4}$ S. Gangopadhayay, G. S. Hadjipanayis, B. Dale, C. M. Sorenson, K. J. Klabunde, V. Papaefthymiou, and A. Kostikas, Phys. Rev. B 45, 9778 (1992).

${ }^{5}$ B. D. Cullity, Introduction to Magnetic Materials (Addison-Wesley, Reading, MA, 1972), pp. 410-418.

${ }^{6}$ S. Mørup and H. Topsøe, Appl. Phys. 11, 63 (1976). 